\title{
Análise da Competitividade da Produção de Soja nos Sistemas de Plantio Direto e Plantio Convencional na Região do Cerrado Brasileiro
}

\author{
Maria Isabel da Silva Azevedo Alvim ${ }^{1}$ \\ Sonia Maria Leite R. do Valle ${ }^{2}$ \\ João Eustáquio Lima ${ }^{3}$ \\ Orlando Monteiro da Silva ${ }^{4}$
}

Resumo - O setor agrícola nacional, em fase da reorganização e adequação aos novos padrões de produção e comercialização, enfrenta o desafio de crescer de modo competitivo e sustentável, para atender a demanda interna e conquistar e manter espaço de mercado externo, fornecendo produtos e processos de qualidade, com sustentabilidade e a preços competitivos. Para atender as novas demandas de uma agricultura mais equilibrada, ecológica e econômica, é necessário a prática de uma produção coerente com o desenvolvimento sustentável, o que o sistema de plantio direto desde a década de 60 vem demonstrando no País.

Assim sendo, é objetivo deste trabalho analisar, utilizando a Matriz de Análise de Políticas, a lucratividade e competitividade da produção de soja no sistema de plantio direto, na região do cerrado de Mato Grosso do Sul que, como se pode observar pelos resultados obtidos, foi maior do que a do sistema de plantio convencional.

Palavras-chaves: Produção e Comercialização Agrícola, Desenvolvimento Sustentável.

\footnotetext{
${ }^{1}$ Prof $^{\text {a }}$ Dr $^{\text {a }}$ FEA/UFJF - E-mail:mialvim@terra.com.br

${ }^{2}$ Profa Pós Dr./UFV - E-mail: smleite@ufv.br

${ }^{3 \text { e } 4}$ Prof $^{\text {os }}$ Dros/UFV - E-mail: jelima@ufv.br / E-mail: odasilva@ufv.br
} 
Abstract - The national agricultural section, in phase of reorganization and adaptation to the new production and sale standards, faces the challenge of growing in a competitive and sustainable way, to meet the internal demand and to conquer and to maintain foreign market space, supplying products and quality processes with sustainability and at competitive prices. To meet the new demands of a more ecologically and economically balanced agriculture, it requires the practice of a production consistent with the sustainable development.

Key-Word - agricultural production and marketing, sustainable development.

Jel Classification: Q21

\section{1 - Introdução}

A globalização recente da economia brasileira, com grande mobilidade de capital e transformações nas estruturas produtivas tradicionais, tem causado importantes modificações em diferentes setores. O país passou a fazer parte de um espaço integrado globalmente e bastante competitivo, provocando avanços tecnológicos e novos métodos de produção e de produtos, oriundos de cadeias produtivas integradas globalmente e onde a questão ambiental passa a ter importância fundamental nos processos de produção, comercialização e consumo. O crescimento acelerado de transações de bens que incorporam inovações tecnológicas e práticas ecologicamente corretas altera substancialmente as vantagens competitivas de vários segmentos econômicos em todos os países.

A maior parte das áreas agrícolas do Brasil são sensíveis à erosão e são de rápida degradação sob as ações do vento, chuva e sol quando cultivadas no sistema convencional. São solos que exigem alta tecnologia para oferecem condições a múltiplas culturas. Em algumas regiões do País, são possíveis até três culturas por ano, mas quando o cultivo acontece no sistema convencional, a base produtiva pode se degradar em poucos anos.

Dentro da análise econômica, a erosão leva a um aumento dos custos de produção das culturas, requerendo gastos adicionais para 
repor a fertilidade do solo, para a adoção de técnicas de conservação, dentre outras.

O sistema de plantio direto - SPD - tem demonstrado sua eficácia na solução dos problemas de solo, primeiramente, com a introdução de práticas de cobertura de solo no inverno e a rotação de culturas, sendo que os testes feitos na cobertura de inverno comprovaram seus efeitos nas lavouras de verão. Avaliar os efeitos da eficácia e eficiência do SPD como contribuição decisiva para uma agricultura sustentável em termos ambientais, economicamente competitiva e socialmente eqüitativa, tem sido o foco de atenção de pesquisadores e produtores, dentro do que se pode denominar "cadeia de sustentabilidade" da agricultura brasileira.

Neste contexto é objetivo deste trabalho analisar a produtividade, lucratividade e competitividade da produção de soja na região, comparando-se os sistemas de plantio direto e plantio convencional.

Especificamente pretende-se determinar e comparar a lucratividade privada e social da produção de soja pelo uso dos sistemas de plantio direto e plantio convencional, bem como analisar os efeitos de alterações nas taxas de câmbio e nos fatores de conversão sobre tal lucratividade.

A região analisada, Mato Grosso do Sul, se justifica por ser, na área de cerrado, uma das últimas a adotar o sistema de plantio direto, devido à expansão de sua área cultivada com soja ocorrer primeiro em solos considerados mais férteis e, assim, o efeito de maior competitividade e lucratividade, como o aproveitamento melhor dos recursos naturais pelo sistema de plantio direto, pode ser melhor verificado.

\section{2 - Metodologia}

A análise desenvolvida neste trabalho teve como suporte teórico os conceitos econômicos de lucratividade, custos sociais e privados de fatores, competitividade de sistemas de produção e política comercial. Os princípios analíticos desses conceitos baseiam-se na Teoria Neoclássica da Firma e Teoria do Comércio Internacional. O instrumental utilizado para essa análise foi a Matriz de Análise de Política (MAP), desenvolvida por MONKE \& PEARSON (1989). 
Análise da Competitividade da Produção de Soja nos Sistemas de Plantio Direto e Plantio Convencional na Região do Cerrado Brasileiro

CARDOSO e BARROS (2002) ressaltam que não há na teoria econômica neoclássica uma definição para competitividade, sendo este um conceito político, ou seja, não há, na economia geral, uma teoria sobre a competitividade porque este não é um termo estritamente econômico. Geralmente, a competitividade é tida como sendo o resultado dos efeitos combinados de distorções de mercado e de vantagens comparativas. As distorções de mercado incluiriam tanto as causadas pela política econômica quanto pela competição imperfeita entre as firmas. Em conseqüência, encontram-se na literatura os mais diferentes conceitos e indicadores para mensurar competitividade. A produtividade e a lucratividade são variáveis importantes na busca de competitividade.

Quando se fala em produtividade, é inerente pensar em rentabilidade, eficiência, tecnologia, inovação e condições de trabalho. A tecnologia compreende o estudo e a racionalização da produção, vinculados especialmente aos processos e métodos que transformam os insumos em produtos. O valor da tecnologia está relacionado com a aplicação que se faz dela para gerar riquezas ou melhorar a qualidade de vida.

A abordagem analítica deste trabalho baseou-se no modelo desenvolvido por MONKE \& PEARSON (1989) de estrutura organizacional denominado Matriz de Análise de Políticas (MAP), originalmente desenvolvida em 1981 como instrumental de análise na política agrícola de Portugal, e cuja atenção é dirigida para padrões eficientes de produção e preço que permitem obter uma avaliação dos efeitos de novas tecnologias sobre a lucratividade do sistema utilizado, através de comparações e variações nos orçamentos gerados pela alteração de uma subsérie de dados de insumos e de produção. Essas comparações proporcionam mais informações quanto à existência ou não de incentivos econômicos para promover a mudança tecnológica. Desde o trabalho pioneiro em Portugal, várias pesquisas seguiram esta mesma metodologia, destacando-se o trabalho desenvolvido pela EMBRAPA na análise de cadeias produtivas no Brasil, na qual os autores utilizam a MAP para definir a competitividade dos produtos analisados. A técnica de análise das cadeias consistiu na aplicação da matriz de Análise de Políticas, que considera custos sociais e privados na produção, no transporte e no beneficiamento ao longo da cadeia, permitindo, com isso, uma análise de eficiência econômica e de competitividade interna e externa (VIEIRA, 2001). 
MONKE e PEARSON (1992) utilizam esta mesma metodologia (MAP) em trabalhos de análise da situação agrícola em países da África. A MAP foi utilizada para analisar a política agrícola no Kenya, em 1995 (PEARSON et al, 1995).

A MAP utiliza dois sistemas contábeis distintos em que se consideram respectivamente os preços de mercado (ou preços privados) e os preços sociais dos diferentes insumos e produtos. A estrutura da matriz permite estimar, com razoável grau de exatidão, os custos e os lucros da produção, revelando a maneira pela qual as políticas afetam a rentabilidade privada e a social de uma atividade. Os itens que compõem as planilhas são: insumos fixos, custo do trabalho, insumos intermediários, outros custos, custo total da produção agrícola, receita com produto e subproduto, lucro antes dos impostos, impostos diretos e lucro após o imposto.

Tabela 1 - Matriz de Análise de Políticas - MAP

\begin{tabular}{l|c|c|c|c}
\hline \multirow{2}{*}{ Discriminação } & \multirow{2}{*}{ Receita } & \multicolumn{2}{|c|}{ Custos } & \multirow{2}{*}{ Lucro } \\
\cline { 3 - 5 } & & $\begin{array}{c}\text { Insumos } \\
\text { Comercializáveis }\end{array}$ & $\begin{array}{c}\text { Fatores } \\
\text { Domésticos }\end{array}$ & \\
\hline Preços Privados & $\mathrm{A}$ & $\mathrm{B}$ & $\mathrm{C}$ & $\mathrm{D}$ \\
\hline Preços Sociais & $\mathrm{E}$ & $\mathrm{F}$ & $\mathrm{G}$ & $\mathrm{H}$ \\
\hline $\begin{array}{l}\text { Efeitos de Divergências } \\
\text { e Eficiência Política }\end{array}$ & $\mathrm{I}$ & $\mathrm{J}$ & $\mathrm{K}$ & $\mathrm{L}$ \\
\hline
\end{tabular}

Lucros Privados - (LP) D = A - B - C

Lucros Sociais - (LS): $\mathrm{H}=\mathrm{E}-\mathrm{F}-\mathrm{G}$

Transferências associadas à produção: $\mathrm{I}=\mathrm{A}-\mathrm{E}$

Transferências associadas ao custo dos insumos comercializáveis: $\mathrm{J}=$ $\mathrm{B}-\mathrm{F}$

Transferências associadas ao custo dos fatores domésticos: $\mathrm{K}=\mathrm{C}-\mathrm{G}$ Transferências líquidas de Políticas: $\mathrm{L}=\mathrm{D}-\mathrm{H}$ ou $\mathrm{L}=\mathrm{I}-\mathrm{J}-\mathrm{K}$. A lucratividade é expressa da seguinte maneira: $D=A-B-C$ Sendo: $A=P^{d} Q^{d}$ Em que $A$ é a receita privada, sendo $P^{d}$ o preço do produto e $\mathrm{Q}^{\mathrm{d}}$ a quantidade total privada de determinado produto: $B=\sum_{i=1}^{n} p_{i}^{d} q_{i}^{d} \begin{aligned} & \text { Em que B é o custo dos insumos comercializáveis, } P_{i}^{d} \text { o } \\ & \text { preço privado do insumos i e } q_{i}^{d} \text { a quantidade privada } \\ & \text { do insumo i utilizado do bem sendo analisado: }\end{aligned}$ 
$C=\sum_{j=i}^{n} w_{j}^{d} l_{j}^{d} \begin{aligned} & \text { C é o custo dos insumos domésticos, em que } w_{j}^{d} \text { é o } \\ & \text { insumo j utilizado. }\end{aligned}$

Os preços privados de mercado incorporam os efeitos de todas as políticas e imperfeições de mercado.

A primeira linha da matriz apresenta os cálculos da lucratividade privada (D) que indicam a competitividade do sistema de produção no período base, para determinado nível tecnológico, dados os valores dos produtos, os custos dos insumos e as políticas de transferências (como exemplo, impostos e subsídios) prevalecentes. Neste caso, o termo competitividade representa resultados financeiros na presença de efeitos de políticas, e/ou imperfeições de mercado. Os resultados financeiros positivos (lucratividade) indicam que o sistema produtivo é competitivo dado as condições existentes.

A segunda linha da matriz apresenta os valores sociais. Nesta linha, a lucratividade é calculada para avaliar a eficiência do sistema de produção agrícola. O conceito de vantagem comparativa é aplicado como medida de lucratividade social ou econômica, ou seja, a medida de vantagem comparativa indica a eficiência de alocação de recursos nacionais (ALVES, 2002). Portanto, a eficiência é obtida quando os recursos de uma economia são utilizados em atividades que proporcionam os maiores níveis de produção e renda. Desse modo, os lucros sociais $(\mathrm{H})$ são uma medida de eficiência, desde que as receitas (E) e os custos de insumos $(F+G)$ sejam avaliados em preços que refletem o custo de oportunidade social. O lucro social é dado por: $\mathrm{H}+\mathrm{E}-\mathrm{F}-\mathrm{G}$

Sendo: $E=P^{s} Q^{s}$ Em que E é a receita social, $P^{s}$ o preço social do produto e $\mathrm{Q}^{\mathrm{s}}$ a quantidade total do produto:

$F=\sum_{i=1}^{n} p_{i}^{s} q_{i}^{s} \begin{aligned} & \text { Sendo F o custo dos insumos comercializáveis, } P_{i}^{s} \text { o pre- } \\ & \text { ço do insumo i e } q_{i}^{s} \text { a quantidade do insumo utilzado; }\end{aligned}$ $G=\sum_{j=1}^{n} w_{j}^{s} l_{j}^{s} \begin{aligned} & \text { Em que } \mathrm{G} \text { é o custo dos insumos domésticos, } w_{j}^{s} \\ & \text { j utilizado. }\end{aligned}$ 
Para produção (E) e insumos (F), que são comercializados mundialmente, considera-se que as avaliações sociais apropriadas são dadas pelos preços internacionais - preço de importação CIF para bens ou serviços que são importados ou preços de exportação FOB para os exportáveis. Considera-se que, a esses preços internacionais, os consumidores e produtores podem importar, exportar ou produzir bens e serviços domesticamente. O valor social da produção doméstica adicional compreende as reservas estrangeiras que não são despendidas pela redução de importações, bem como o valor das reservas ganhos pela expansão das exportações (para cada unidade de produção, o preço de importação CIF ou de exportação FOB).

Como medida de eficiência ou vantagem comparativa, o lucro social $\mathrm{H}=(\mathrm{E}-\mathrm{F}-\mathrm{G})$, quando negativo, indica que o sistema não é considerado economicamente viável no contexto de mercado internacional, sem assistência do governo. Tem-se uma indicação de que tal sistema não assegura a alocação economicamente eficiente de recursos escassos.

A terceira identidade - I, J, K e L, refere-se às diferenças entre os valores privados e sociais de receitas, custos e lucros. Para cada entrada na matriz - mensurada verticalmente - uma eventual diferença entre o preço privado observado (mercado doméstico) e o preço social estimado (eficiência) deve ser atribuído aos efeitos de políticas (na forma de taxação, subsídios, restrições comerciais e distorções na taxa de câmbio) ou pela existência de falhas de mercados de produtos e de fatores. Essa relação é originada diretamente da definição de preço social (ALVES, 2002).

Na tabela 2, pela análise da MAP expandida, cada entrada mede os efeitos de divergência e eficiência política (I, J, K e L) e é desagregada em três categorias:

Falhas de mercado (M, N, O, P); Políticas distorcidas (Q, R, S, T) e Políticas eficientes (U, V, W, X). 
Tabela 2 - Expansão da Matriz de Análise de Políticas - MAP

\begin{tabular}{|l|c|c|c|c|}
\hline \multirow{2}{*}{ Discriminação } & \multirow{2}{*}{ Receita } & \multicolumn{2}{|c|}{ Custos } & \multirow{2}{*}{ Lucro } \\
\cline { 3 - 4 } & & Ins. Comerc. & Fat. Domest. & \\
\hline Preços Privados & $\mathrm{A}$ & $\mathrm{B}$ & $\mathrm{C}$ & $\mathrm{D}$ \\
\hline Preços Sociais & $\mathrm{I}$ & $\mathrm{F}$ & $\mathrm{G}$ & $\mathrm{H}$ \\
\hline $\begin{array}{l}\text { Divergências e } \\
\text { Efic. Política }\end{array}$ & $\mathrm{M}$ & $\mathrm{N}$ & $\mathrm{K}$ & $\mathrm{L}$ \\
\hline $\begin{array}{l}\text { Imperfeições de Mercado } \\
\text { Efeitos de Distorções } \\
\text { Políticas }\end{array}$ & $\mathrm{Q}$ & $\mathrm{R}$ & $\mathrm{O}$ & $\mathrm{P}$ \\
\hline $\begin{array}{l}\text { Efeitos de Política } \\
\text { Eficiente }\end{array}$ & $\mathrm{U}$ & $\mathrm{V}$ & $\mathrm{W}$ & $\mathrm{T}$ \\
\hline
\end{tabular}

Fonte: MONKE \& PEARSON (1989)

Lucros Privados (LP): D = A - B - C

Lucros sociais (LS): $\mathrm{H}=\mathrm{E}-\mathrm{F}-\mathrm{G}$

Transferências associadas à produção: $\mathrm{I}=\mathrm{A}-\mathrm{E}=\mathrm{M}+\mathrm{Q}+\mathrm{U}$

Transferências associadas ao custo insumos comercializáveis: $\mathrm{J}=\mathrm{B}-$ $\mathrm{F}=\mathrm{N}+\mathrm{R}+\mathrm{V}$

Transferências associadas ao custo dos fatores: $\mathrm{K}=\mathrm{C}-\mathrm{G}=\mathrm{O}+\mathrm{S}+$ W

Transferências líquidas: $\mathrm{L}=\mathrm{D}-\mathrm{H}=\mathrm{I}-\mathrm{J}-\mathrm{K}=\mathrm{P}+\mathrm{T}+\mathrm{X}$.

A mensuração das transferências ${ }^{3}$ associadas à produção pode ser representada pela expressão: $\mathrm{I}=\mathrm{A}-\mathrm{E}=\mathrm{M}+\mathrm{Q}+\mathrm{U}$

E as transferências associadas aos custos de insumos comercializáveis ( $\mathrm{J}$ ) são dados por: $\mathrm{J}=\mathrm{B}-\mathrm{F}=\mathrm{N}+\mathrm{R}+\mathrm{V}$

Considera-se que essas transferências resultam de dois tipos de políticas que causam divergência entre os preços domésticos dos produtos e os preços internacionais, caracterizadas como políticas específicas de produtos e política cambial.

Os custos sociais de fatores $(G)$ refletem condições de oferta e demanda subordinados aos mercados de fatores domésticos. Os preços de fator são, desse modo, influenciados pelo conjunto prevalecente de

\footnotetext{
${ }^{3}$ Considerando que as transferências decorrentes dos efeitos de políticas ocorrem no sentido dos produtores para os consumidores ou vice-versa.
} 
políticas macroeconômicas e de preço de produto. A atuação do governo pode ainda criar divergências entre custos privados (C) e custos sociais (G) por intermédio de política tributária ou de subsídios para um ou mais fatores de produção (capital, trabalho e terra).

As falhas de mercado, decorrentes de informações imperfeitas ou de desenvolvimento ineficiente das instituições que consistem em características do funcionamento de mercados, podem também influenciar os preços dos produtos e de fatores. Considerando que existem imperfeições de mercado de fator juntamente com políticas de fator “distorcivas", tem-se que ambos, O e S, e possivelmente W, são componentes positivos das transferências associadas ao custo dos fatores domésticos (K). Essas transferências são representadas como: $\mathrm{K}=\mathrm{C}$ $-\mathrm{G}=\mathrm{O}+\mathrm{S}+\mathrm{W}$

Dessa forma, a transferência líquida (L) combina efeitos de políticas “distorcivas” (I, J e S que são componente de K) com aqueles de falhas de mercado de fator (representado por O, componente de $\mathrm{K}$ ) e políticas eficientes para compensá-los (representado por $\mathrm{W}$, componente de $\mathrm{K})$. A transferência líquida pode ser representada como:

$$
\mathrm{L}=\mathrm{D}-\mathrm{H}=\mathrm{P}+\mathrm{T}+\mathrm{X}
$$

Através de uma comparação de lucros privados e sociais, pode-se encontrar a transferência líquida. Essas medidas de transferência líquida devem, por definição, ser idênticas na matriz de contabilidade de dupla entrada, $\mathrm{L}=(\mathrm{I}-\mathrm{J}-\mathrm{K})=(\mathrm{D}-\mathrm{H})$. A desagregação da transferência líquida total indica que cada política "distorciva” fornece transferências positivas ou negativas para o sistema. Assim, a MAP permite a comparação dos efeitos de falhas de mercado e políticas "distorcivas" para o grupo inteiro de políticas de produtos e de "macropreço", assim considerados a taxa de câmbio e os preços de fatores, como a taxa de juros, salários e aluguel da terra. Mudanças nesses preços alteram os preços de insumos e produtos e, dessa forma, influenciam a lucratividade agrícola.

Os indicadores privados e sociais são obtidos das relações em que ambos os numeradores e denominadores de cada relação são extraídos da MAP em unidades de moeda nacional por unidade física do bem. Essas relações são as seguintes:

Razão do Custo Privado (RCP) calculada para proporcionar uma 
medida mais precisa de competitividade. Os resultados da lucratividade privada, que indicam a competitividade sob as condições de políticas existentes, são residuais e poderiam ter vindo de sistemas, utilizando níveis muito diferentes de insumos e de capital. Tal problema pode ser contornado pela construção de um indicador de custo privado RCP, que é a razão entre os custos dos fatores domésticos a preços privados (C) e o valor adicionado a preços privados $(A-B)$, isto é, $\mathrm{RCP}=\mathrm{C} /(\mathrm{A}-\mathrm{B})$

Este indicador mostra quanto de renda o sistema de produção pode gerar para pagar os fatores domésticos e, ainda, permanecer competitivo, isto é, não ter lucros nem prejuízos, após obter lucros normais, em que $(A-B-C)=D=0$ e o RCP $=1$.

A razão do custo privado pode indicar ainda se os fatores domésticos estão recebendo o seu retorno normal $(\mathrm{RCP}=1)$, acima do retorno normal $(\mathrm{RCP}<1)$ ou abaixo do retorno normal (RCP > 1) (ALVES, 2002).

O custo dos recursos domésticos (CRD) é uma medida de vantagem comparativa mais criteriosa que a lucratividade social; a razão é a mesma pela qual a RCP é mais precisa que a lucratividade privada, só que utilizando os valores sociais. Como indicador de vantagem comparativa (eficiência), o CRD indica o comportamento da lucratividade social, ou seja, quanto se despende de recursos domésticos em valores sociais para gerar uma unidade de divisas por meio de exportação, definido pela razão entre o valor social dos recursos domésticos $(G)$ e o valor adicionado $(E-F)$ expresso em equivalente social, isto é:

$$
\mathrm{CRD}=\mathrm{G} /(\mathrm{E}-\mathrm{F})
$$

O CPN indica o grau de transferência implícita no preço do produto, por exemplo, um CPN de 1,1 indica que prevalecem políticas que estão incrementando o preço de mercado em um nível de $10 \%$ maior que o preço internacional. Interpreta-se o CPN como um indicador do grau de proteção ou desproteção (interpretado no sentido de que os efeitos das políticas são de beneficiar ou não os valores privados dos produtos, e/ou insumos), dado aos sistemas produtivos. Quando esse coeficiente é menor do que 1, tem-se uma indicação de que os produtores do bem estariam sendo penalizados, à medida que recebem pre- 
ços inferiores ao que pode ser considerado como nível de preço de eficiência.

Insumos comercializados internacionalmente estão sujeitos a tarifas, impostos ou subsídios. Nestas circunstâncias, o centro da análise desloca da proteção ao produto final para a proteção que o sistema tributário concede ao processo produtivo. Define-se, então, a taxa de proteção efetiva como taxa de proteção ao valor adicionado em dado processo produtivo. Uma atividade qualquer só é verdadeiramente protegida se o resultado líquido da combinação da estrutura protecionista com os ajustamentos cambiais age no sentido de elevar o valor adicionado naquela atividade. O coeficiente de proteção efetiva consiste na razão entre o valor adicionado doméstico a preços de mercado e este valor a preços internacionais (ALVES, 2002).

$\mathrm{CPE}=(\mathrm{A}-\mathrm{B}) /(\mathrm{E}-\mathrm{F}) \mathrm{O}$ coeficiente dessa razão mede o grau de transferências entre produtores e a sociedade derivadas de políticas que afetam o mercado de produto e insumos comercializáveis. Assim, é possível determinar quanto as políticas afastam os preços dos produtos e dos insumos de seus preços de eficiência. Valores desse coeficiente menores do que 1 indicam que há desproteção ao produtor agrícola, uma vez que o valor adicionado na produção é inferior ao valor adicionado em termos econômicos. A transferência das políticas sobre o produto e sobre o insumo comercializáveis, tomadas em conjunto, é negativa sob a perspectiva do produtor que recebe e paga em valores privados. Os valores maiores que 1 indicam um resultado oposto, ou seja, os produtores são favorecidos.

O coeficiente de lucratividade - CL - é uma extensão do CPE, que inclui as transferências entre os produtores e consumidores (sociedade) decorrentes dos efeitos de políticas sobre os fatores domésticos. O CL é a razão entre lucratividade privada e social, expresso como: $\mathrm{CL}=$ $(\mathrm{A}-\mathrm{B}-\mathrm{C}) /(\mathrm{E}-\mathrm{F}-\mathrm{G})=\mathrm{D} / \mathrm{H}$

A razão de subsídios à produção (RSP) é o indicador final de incentivos. Final no sentido de ser conclusivo em termos de medida e também abranger os efeitos de políticas sobre toda a produção. É uma medida de transferência líquida de políticas (L) como uma proporção das receitas sociais totais (E). A expressão desse indicador assume a seguinte forma: $\mathrm{RSP}=\mathrm{L} / \mathrm{E}=(\mathrm{D}-\mathrm{H}) / \mathrm{E}$ 
Análise da Competitividade da Produção de Soja nos Sistemas de Plantio Direto e Plantio Convencional na Região do Cerrado Brasileiro

\section{3 - Resultados e Discussão}

São apresentados a seguir, os resultados da análise realizada para a produção de soja no Estado de Mato Grosso do Sul, empregando-se os sistemas de plantio direto (SPD) e plantio convencional (SPC), para a safra agrícola de 2001.

Todos os valores estão em reais (R\$), de setembro de 2001, sendo as variáveis analisadas, os valores calculados para as lucratividades privada e social, os efeitos de divergências e eficiência de políticas, bem como os indicadores privados e sociais.

Tabela 3 - Matriz de Análise de Política - MAP - produção de soja, MS, 2001. SPD (ha)

\begin{tabular}{|l|c|c|c|c|}
\hline \multirow{2}{*}{ Itens } & \multirow{2}{*}{ Receita } & \multicolumn{2}{|c|}{ Custos } & \multirow{2}{*}{ Lucro } \\
\cline { 3 - 4 } & & $\begin{array}{c}\text { Insumos } \\
\text { Comercializáveis }\end{array}$ & $\begin{array}{c}\text { Fatores } \\
\text { Domésticos }\end{array}$ & \\
\hline \multirow{2}{*}{ Valores Privados } & $(\mathrm{A})$ & $(\mathrm{B})$ & $(\mathrm{C})$ & $(\mathrm{D})$ \\
\hline & 1050,08 & 413,02 & 370,57 & 266,49 \\
Valores Sociais & $(\mathrm{E})$ & $(\mathrm{F})$ & $(\mathrm{G})$ & $(\mathrm{H})$ \\
\hline Efeitos de Divergências & 1218,09 & 361,98 & 370,11 & 486,00 \\
e Eficiência Política & $-168,01$ & 51,04 & $(\mathrm{~K})$ & $(\mathrm{L})$ \\
\end{tabular}

Fonte: dados da pesquisa.

Tabela 4 - Matriz de Análise de Política - MAP - produção de soja, MS, 2001. SPC (ha)

\begin{tabular}{|c|c|c|c|c|}
\hline \multirow[t]{2}{*}{ Itens } & \multirow[t]{2}{*}{ Receita } & \multicolumn{2}{|c|}{ Custos } & \multirow[t]{2}{*}{ Lucro } \\
\hline & & $\begin{array}{c}\text { Insumos } \\
\text { Comercializáveis }\end{array}$ & $\begin{array}{c}\text { Fatores } \\
\text { Domésticos }\end{array}$ & \\
\hline Valores Privados & $\begin{array}{c}\text { (A) } \\
1050,08\end{array}$ & $\begin{array}{c}\text { (B) } \\
415,70\end{array}$ & $\begin{array}{c}\text { (C) } \\
415,50\end{array}$ & $\begin{array}{c}\text { (D) } \\
218,88\end{array}$ \\
\hline Valores Sociais & $\begin{array}{c}\text { (E) } \\
1218,09\end{array}$ & $\begin{array}{c}(\mathrm{F}) \\
367,75\end{array}$ & $\begin{array}{c}\text { (G) } \\
404,44\end{array}$ & $\begin{array}{c}(\mathrm{H}) \\
445,90\end{array}$ \\
\hline $\begin{array}{l}\text { Efeitos de Divergências } \\
\text { e Eficiência Política }\end{array}$ & $\begin{array}{c}\text { (I) } \\
-168,01\end{array}$ & $\begin{array}{c}(\mathrm{J}) \\
47,95\end{array}$ & $\begin{array}{c}(\mathrm{K}) \\
11,06\end{array}$ & $\begin{array}{c}(\mathrm{L}) \\
-227,02\end{array}$ \\
\hline
\end{tabular}

Fonte: dados da pesquisa. 


\section{1 - Lucratividade Privada e Social}

Os resultados apresentados sobre lucratividade, de $\mathrm{R} \$ 266,49$ (SPD) e $\mathrm{R} \$ 218,88$ (SPC), por hectare, indicam a competitividade dos sistemas analisados, do ponto de vista privado, por apresentarem lucros positivos. Indicam ainda, que a produção de soja na região é competitiva e lucrativa, mesmo sob as condições atuais das políticas públicas.

As lucratividades sociais positivas indicam que há eficiência econômica nos dois sistemas e que há eficiência na geração de divisas e na alocação de recursos naturais nacionais

Os valores do SPD mostram sua maior eficiência e competitividade não só para obtenção de menores custos de produção, quanto também na conservação e preservação dos recursos naturais.

\section{2 - Transferências Associadas à Produção}

As transferências associadas à produção de soja, nos SPD e SPCP, apresentaram o montante negativo de $\mathrm{R} \$ 168,01$.

A soja, como um dos principais produtos de exportação do Brasil, tem seu desempenho, de aumento ou retração da produção, atrelado ao desempenho do mercado mundial.

Comparando-se os preços praticados no mercado de Mato Grosso do Sul e os preços internacionais de soja, observa-se que há uma transferência negativa nos dois sistemas de produção, da ordem de aproximadamente $17 \%$. Isto significa que para cada hectare produzido, o produtor local, recebe menos do que ele poderia receber caso políticas de taxação à produção (ICMS e outros impostos) não tivessem sido implementadas.

\section{3 - Transferências Associadas aos Custos de Produção}

Os cálculos associados aos custos dos insumos comercializáveis indicam que as transferências são positivas no montante de $\mathrm{R} \$ 51,04$ para o SPD e R $\$ 47,95$ para o SPC.

Em relação aos insumos comercializáveis, os efeitos das divergências entre as avaliações sociais e privadas, são atribuídos às políticas 
distorcivas, e não às falhas de mercado. Essas políticas fazem com que os preços de mercado (privado) destes insumos, difiram dos preços mundiais (sociais).

A análise das transferências associadas aos custos dos insumos comercializáveis para a soja, indica que o governo tem adotado políticas intervencionistas no mercado deste produto, modificando as relações entre as avaliações privadas e sociais dos custos dos insumos comercializáveis, e a soja seria mais competitiva se fossem reduzidas ou mesmo eliminadas certas alíquotas de importação de insumos.

Os valores positivos representam custos privados maiores que os custos sociais dos fatores domésticos, mostrando transferência negativa à produção de soja, já que contribui para uma redução nos lucros privados. Os valores apresentados mostram melhor situação para o SPD.

\section{4 - Indicadores Privados e Sociais}

Os indicadores privados e sociais permitam comparações entre os sistemas de produção analisados, possibilitando uma melhor avaliação desses sistemas diante das limitações econômicas impostas e principalmente em relação à melhor alocação de recursos.

Tabela 5 - Coeficientes da lucratividade dos SPD e SPC de produção de soja. MS, 2001.

\begin{tabular}{lll}
\hline Indicadores Privados e Sociais & SPD & SPC \\
\hline $\begin{array}{l}\text { 1 - Razão do Custo Privado } \\
\text { RCP = C / (A - B) }\end{array}$ & 0,58 & 0,65 \\
$\begin{array}{l}\text { - Custo dos Recursos Domésticos } \\
\text { CRD = G / (E - F) }\end{array}$ & 0,43 & 0,48 \\
$\begin{array}{l}3-\text { Coeficiente de Proteção Nominal } \\
\text { CPN = A / E }\end{array}$ & 0,86 & 0,86 \\
$\begin{array}{l}4-\text { Coeficiente de Proteção Efetiva } \\
\text { CPE = (A - B) / (E - F) }\end{array}$ & 0,74 & 0,75 \\
$\begin{array}{l}\text { C - Coeficiente de Lucratividade } \\
\text { CL = D / H }\end{array}$ & 0,55 & 0,49 \\
$\begin{array}{l}\text { R - Razão do Subsídio } \\
\text { RS = L / E }\end{array}$ & & \\
\hline
\end{tabular}

Fonte: dados da pesquisa. 
A razão do custo privado (RCP) é um indicador que mostra quanto o sistema produtivo pode produzir para pagar os fatores domésticos, e ainda permanecer competitivo.

Os resultados apresentados, de 0,58 para o SPD e 0,65 para o SPC, em preços privados menores do que 1 , indicam que os fatores domésticos estão recebendo acima do retorno normal, ou seja, são lucrativos do ponto de vista econômico.

Mesmo ambos os sistemas sendo lucrativos, o SPD forneceu um indicador de custo privado $8 \%$ menor do que o SPC, o que lhe confere maior potencial de expansão.

O custo do recurso doméstico (CRD) é calculado para avaliar o comportamento da lucratividade social dos sistemas e é analisado de forma análoga à da lucratividade privada, ou seja, minimizar o CRD é equivalente a maximizar os lucros sociais. Mede-se aqui a eficiência do sistema de produção ou sua vantagem competitiva.

Os resultados de 0,43 para SPD e 0,48 para SPC, inferiores à unidade, indicam que os valores sociais dos recursos domésticos empregados na produção de soja são inferiores aos valores sociais adicionados. A utilização de recursos domésticos para gerar uma unidade monetária de divisas por meio de exportação é menor que a unidade, indicando que $\mathrm{R} \$ 0,43$ no SPD e R $\$ 0,48$ no SPC, em recursos domésticos, seriam suficientes para gerar $\mathrm{R} \$ 1,00$ de divisas por meio da exportação, o que significa que a expansão dessa atividade representa ganhos líquidos para o País, considerando-se, em termos de eficiência econômica, a locação de recursos.

O CPN indicou que o produtor de soja em MS está recebendo menos que no mercado internacional, ou seja, como este coeficiente indica proteção ou desproteção das políticas públicas, seu resultado mostrou que os produtores, embora em um volume pequeno, estão sendo penalizados por receberem preços inferiores ao que poderia ser como nível de preço de eficiência. O coeficiente igual para os dois sistemas indica que o uso da tecnologia de produção, neste fator, não teve influência nos níveis de desproteção registrados.

O CPE - coeficiente de proteção efetiva - é um indicador que combina os efeitos dos indicadores anteriores a fim de verificar a extensão dos incentivos que os sistemas de produção recebem das políticas go- 
Análise da Competitividade da Produção de Soja nos Sistemas de Plantio Direto e Plantio Convencional na Região do Cerrado Brasileiro

vernamentais. Estima o quanto as políticas que afetam o mercado de produtos fazem o valor adicionado diferir do valor que ocorreria na sua ausência. Os coeficientes aqui registrados de 0,74 para o SPD e de 0,75 para o SPC indicam praticamente a mesma desproteção nos dois sistemas, aos fatores de produção.

O coeficiente de lucratividade (CL) é a razão entre o lucro privado e o lucro social. Dimensiona a distância entre o lucro privado e o lucro que se obteria na ausência de políticas causadoras de distorção. É considerado um indicador completo na proteção alcançada pelas políticas vigentes, pois leva em conta os efeitos de transferência de políticas no mercado de fatores. Mede o efeito global de todas as políticas. Os valores dos CLs de 0,55 para SPD e 0,49 para o SPC, indicam que a produção de soja está sendo liquidamente taxada, ou seja, indicam desproteção à atividade.

A razão de subsídio ao produtor (RS) é a transferência líquida de política como proporção da receita econômica total. Permite comparações sobre a extensão em que as políticas subsidiam os sistemas e pode ser desagregada para mostrar os efeitos de políticas de produtos, insumos e fatores.

Os coeficientes negativos aqui apresentados, de - 0,18 para SPD e 0,19 para SPC, indicam que houve taxação, ou seja, existiu desproteção, provocando redução de $18 \%$ e $19 \%$, respectivamente, na rentabilidade.

\section{4 - Análise de Sensibilidade}

Foi feita ainda, uma análise de sensibilidade no sentido de verificar as conseqüências sobre a produtividade e competitividade da produção de soja, de alterações na taxa de câmbio e nos fatores de conversão, o primeiro por ser considerado um dos mais sujeitos às incertezas, e o segundo no caso de haver erros de estimação.

Os impactos foram analisados sobre os indicadores de razão do custo privado (RCP), custo do recurso doméstico (CRD), coeficiente de proteção nominal (CPN), coeficiente de proteção efetiva (CPE), coeficiente de lucratividade (CL) e razão do subsídio (RS).

A variação na taxa de câmbio utilizada para os preços da soja, expressos em dólar, e dos insumos comercializáveis, foi de um incremen- 
to de $5 \%$ e de $10 \%$, mantendo os demais parâmetros constantes e iguais para os dois sistemas de produção.

A escolha de preços sociais para os produtos e insumos pode levar a uma imprecisão analítica. Isto porque, no processo de valoração social, os fatores de conversão utilizados podem apresentar falhas no seu processo de obtenção, por exemplo, a omissão de informações relevantes de impostos e tarifas. Nesse caso, uma mudança nos fatores de conversão dos valores privados para sociais, pode indicar os efeitos dessa variação sobre os indicadores da MAP. A alteração realizada também foi de $5 \%$ e de $10 \%$.

\section{1 - Variação na Taxa de Câmbio}

Tabela 6 - Análise de sensibilidade dos indicadores da MAP dado um incremento de $5 \%$ e de $10 \%$ na taxa de câmbio(R \$/US\$) para a produção de soja, MS-2001 - SPD e SPC

\begin{tabular}{lcccccc}
\hline Indicadores Privados e Sociais & \multicolumn{3}{c}{ SPD } & \multicolumn{3}{c}{ SPC } \\
& Base & $5 \%$ & $10 \%$ & Base & $5 \%$ & $10 \%$ \\
\hline $1-\mathrm{RCP}=\mathrm{C} /(\mathrm{A}-\mathrm{B})$ & 0,58 & 0,58 & 0,58 & 0,65 & 0,65 & 0,65 \\
$2-\mathrm{CRD}=\mathrm{G} /(\mathrm{E}-\mathrm{F})$ & 0,43 & 0,41 & 0,40 & 0,48 & 0,45 & 0,44 \\
$3-\mathrm{CPN}=\mathrm{A} / \mathrm{E}$ & 0,86 & 0,82 & 0,79 & 0,86 & 0,82 & 0,79 \\
$4-\mathrm{CPE}=(\mathrm{A}-\mathrm{B}) /(\mathrm{E}-\mathrm{F})$ & 0,74 & 0,71 & 0,69 & 0,75 & 0,71 & 0,69 \\
$5-\mathrm{CL}=\mathrm{D} / \mathrm{H}$ & 0,55 & 0,50 & 0,50 & 0,49 & 0,45 & 0,43 \\
$6-\mathrm{RS}=\mathrm{L} / \mathrm{E}$ & $-0,18$ & $-0,20$ & $-0,21$ & $-0,19$ & $-0,21$ & $-0,22$ \\
\hline
\end{tabular}

Fonte: dados da pesquisa

Os resultados alcançados indicam pequenas alterações a um incremento de $5 \%$ na taxa de câmbio e uma maior alteração para $10 \%$ em todos os indicadores, com exceção do indicador de Razão do Custo Privado, como era de se esperar, uma vez que este indicador não sofre interferência dos preços sociais em seu cálculo.

Tais resultados não comprometem o caráter competitivo da produção de soja, sendo que os coeficientes que apresentaram maior sensibilidade são os que dependem, para sua composição, dos valores sociais, 
Análise da Competitividade da Produção de Soja nos Sistemas de Plantio Direto e Plantio Convencional na Região do Cerrado Brasileiro

os quais têm em seus cálculos, a taxa de câmbio como um dos principais componentes.

Verificou-se também, a maior competitividade e lucratividade do SPD, frente ao SPC.

\section{2 - Variação nos Fatores de Conversão}

Os efeitos de variação de $5 \%$ e de $10 \%$ nos fatores de conversão sobre os indicadores da MAP estão apresentados na tabela 7 .

Tabela 7 - Análise de sensibilidade sobre os indicadores da MAP, de um incremento de $5 \%$ e de $10 \%$ nos fatores de conversão sobre a produção de soja, MS, 2001 - SPD e SPC

\begin{tabular}{lccccccc}
\hline Indicadores Privados e Sociais & \multicolumn{3}{c}{$\mathrm{SPD}$} & & \multicolumn{3}{c}{$\mathrm{SPC}$} \\
& Base & $5 \%$ & $10 \%$ & & Base & $5 \%$ & $10 \%$ \\
\hline $1-\mathrm{RCP}=\mathrm{C} /(\mathrm{A}-\mathrm{B})$ & 0,58 & 0,58 & 0,58 & 0,65 & 0,65 & 0,65 \\
$2-\mathrm{CRD}=\mathrm{G} /(\mathrm{E}-\mathrm{F})$ & 0,43 & 0,43 & 0,44 & 0,48 & 0,48 & 0,49 \\
$3-\mathrm{CPN}=\mathrm{A} / \mathrm{E}$ & 0,86 & 0,82 & 0,79 & 0,86 & 0,82 & 0,79 \\
$4-\mathrm{CPE}=(\mathrm{A}-\mathrm{B}) /(\mathrm{E}-\mathrm{F})$ & 0,74 & 0,71 & 0,69 & 0,74 & 0,71 & 0,69 \\
$5-\mathrm{CL}=\mathrm{D} / \mathrm{H}$ & 0,55 & 0,52 & 0,52 & 0,49 & 0,46 & 0,47 \\
$6-\mathrm{RS}=\mathrm{L} / \mathrm{E}$ & $-0,18$ & $-0,18$ & $-0,19$ & $-0,19$ & $-0,19$ & $-0,20$ \\
\hline
\end{tabular}

Fonte: dados da pesquisa

Um incremento de $10 \%$ nos fatores de conversão causa uma alteração um pouco mais significativa do que de $5 \%$ em todos os indicadores, com exceção novamente do coeficiente da razão do Custo Privado, por ser este indicador construído com preços internos, não sofrendo influência dos preços do mercado internacional. Estas variações ocorrem com maior intensidade nos indicadores da produção ao seu maior valor da receita social, a qual depende diretamente do fator de conversão.

Tais resultados, para ambos sistemas de produção, SPD e SPC, mostram que as políticas públicas, embora por vezes tenham reduzido a receita privada em relação à social, não chegam a comprometer a competitividade da produção de soja na região, ou seja, mesmo ocorrendo falhas nas políticas públicas que implicam em aumentos nos custos de produção para o setor agrícola, fica clara a potencialidade deste setor para o crescimento da competitividade nacional. 


\section{5 - Conclusão}

A lucratividade calculada para os dois sistemas de produção, indicou a competitividade da produção de soja para ambas tecnologias, do ponto de vista privado, por apresentarem lucros positivos. O lucro maior dado pelo SPD permite confirmar a preferência por este sistema de produção dado sua maior rentabilidade.A lucratividade social positiva calculada constitui um indicador de que existem vantagens comparativas nos dois sistemas analisados. Isto significa que não há perdas na alocação de recursos, pois está havendo produção com custos sociais inferiores ao custo de importação. O SPD apresentou maiores vantagens comparativas, o que comprova que, além de sua maior lucratividade, este sistema é o mais indicado para se desenvolver uma agricultura sustentável, por ser melhor conservador de recursos naturais.

A MAP permitiu identificar que ambos os sistemas, tanto de plantio direto quanto de plantio convencional, para a produção de soja, na região, são lucrativos e competitivos, no entanto, maiores ganhos, em termos de uso dos recursos disponíveis e de custos de produção podem ser obtidos na SPD. Com isso, esta análise pode subsidiar a tomada de decisão para o sistema de produção a ser utilizado ao nível de produtor, bem como que políticas deveriam ser adotadas pelo governo para obter resultados mais eficazes quanto à produtividade e competitividade do setor agrícola, ou seja, a eficiência econômica ou vantagem comparativa dos sistemas agrícolas.

\section{6- Referências Bibliográficas}

ALVES, J.M. Competitividade e tendência da produção de manga para exportação do nordeste brasileiro. Piracicaba, 2002, 246p. Tese (Doutorado) - ESALQ/USP. Escola Superior de Agricultura Luiz de Queiroz/ Universidade de São Paulo

CARDOSO, C.E.L; BARROS, G.S.C. A quase renda como indicador de competitividade em cadeias agroindustriais: uma proposta a ser implementada na cadeia de fécula de mandioca no Brasil. In: XL Congresso Brasileiro de Economia e Sociologia Rural - Eqüidade e 
eficiência na agricultura brasileira. Passo Fundo/RS. 28 a 31 de julho/02.

MONKE, E \& PEARSON, S. R. Policy Analysis for Agricultural Development. Ithaca and Londo: Cornell University, 1989. 220p. Introduction to the Policy Analysis Matrix. Preparing and Communicating Policy Advice: Aplications of the policy Analysis Matrix to African Agriculture.pp.3 - 11 Seminar Entitled Management for Agricultural Growth in Southern Africa. Harare: Instituto para o desenvolvimento Econômico e Universidade do Zimbabwe.

VIEIRA, R.C.M.T (et. al.) Cadeias produtivas no Brasil: análise de competitividade. Brasília; EMBRAPA, 2001.

PEARSON, SCOTT and ERIC MONKE. Agricultural Policy in Kenya: Aplications of the Policy Analysis Matrix. Ithaca: Cornell University Press, 1995.

Recebido em abril de 2003 e revisto em março de 2004. 